Combined use of transcranial magnetic stimulation and metal electrode implants: a theoretical assessment of safety considerations

This article has been downloaded from IOPscience. Please scroll down to see the full text article.

2012 Phys. Med. Biol. 577813

(http://iopscience.iop.org/0031-9155/57/23/7813)

View the table of contents for this issue, or go to the journal homepage for more

Download details:

IP Address: 128.178.183.146

The article was downloaded on 20/11/2012 at 21:21

Please note that terms and conditions apply. 


\title{
Combined use of transcranial magnetic stimulation and metal electrode implants: a theoretical assessment of safety considerations
}

\author{
Laleh Golestanirad $^{1,2}$, Hossein Rouhani $^{3}$, Behzad Elahi $^{4}$, \\ Kamal Shahim ${ }^{5}$, Robert Chen ${ }^{4}$, Juan R Mosig ${ }^{2}$, Claudio Pollo ${ }^{6}$ \\ and Simon J Graham ${ }^{1}$ \\ ${ }^{1}$ Sunnybrook Research Institute and Department of Medical Biophysics, University of Toronto, \\ Toronto, Canada \\ ${ }^{2}$ Laboratory of Electromagnetics and Acoustics, École Polytechnique Fédérale de Lausanne \\ (EPFL), Lausanne, Switzerland \\ ${ }^{3}$ Rehabilitation Engineering Laboratory, Institute of Biomaterials and Biomedical Engineering, \\ University of Toronto, Toronto, Canada \\ ${ }^{4}$ Toronto Western Research Institute and Department of Neurology, University of Toronto, \\ Toronto Canada \\ ${ }^{5}$ Laboratory of Material Science, École Polytechnique Fédérale de Lausanne (EPFL), Lausanne, \\ Switzerland \\ ${ }^{6}$ Department of Neurosurgery, University Hospital of Bern, Bern, Switzerland \\ E-mail: 1.golestani@gmail.com
}

Received 18 July 2012, in final form 1 October 2012

Published 8 November 2012

Online at stacks.iop.org/PMB/57/7813

\begin{abstract}
This paper provides a theoretical assessment of the safety considerations encountered in the simultaneous use of transcranial magnetic stimulation (TMS) and neurological interventions involving implanted metallic electrodes, such as electrocorticography. Metal implants are subject to magnetic forces due to fast alternating magnetic fields produced by the TMS coil. The question of whether the mechanical movement of the implants leads to irreversible damage of brain tissue is addressed by an electromagnetic simulation which quantifies the magnitude of imposed magnetic forces. The assessment is followed by a careful mechanical analysis determining the maximum tolerable force which does not cause irreversible tissue damage. Results of this investigation provide useful information on the range of TMS stimulator output powers which can be safely used in patients having metallic implants. It is shown that conventional TMS applications can be considered safe when applied on patients with typical electrode implants as the induced stress in the brain tissue remains well below the limit of tissue damage.
\end{abstract}

(Some figures may appear in colour only in the online journal) 


\section{Introduction}

In the past decade dramatic progress in both electrophysiology and bio-electromagnetic theory has occurred with the development of several new techniques to help improve understanding of brain function and brain electrical properties. Induced electrical currents are now used both experimentally and clinically within the human nervous system to influence neural activity and behavior, in both normal and disease states. Among these techniques, transcranial magnetic stimulation (TMS) has emerged as key tool for the non-invasive, painless exploration of cognitive function (Pascual-Leone et al 2000, Wagner et al 2008). The technique has provided researchers and clinicians a versatile means of neural stimulation by using the principles of electromagnetic induction (Barker et al 1987, Ilmoniemi and Ruohonen 1999). Brain excitability state can also be modulated through appropriately timed bursts of TMS, and cortical pathways can be either inhibited or excited by changing the frequency and trains of timed and repeated bursts. These stimulation paradigms are capable of engaging various intracortical pathways or introducing long-term changes in synaptic strength (Classen et al 2004).

TMS alone or in combination with other electrophysiological techniques can be used to assess alterations in the corticomuscular or corticocortical connectivity in various pathologic conditions such as stroke, spinal cord injury, multiple sclerosis, Parkinson's disease and motor neuron disorders (Dimyan and Cohen 2010, Elahi and Chen 2009, Nowak et al 2010, Rossini and Rossi 2007). Pertinent to these applications, TMS has also been suggested as a means of assessing various intracortical inhibitory and excitatory pathways within human motor cortex or between different regions of neocortex (Kujirai et al 1993).

Increasing application of TMS raises the concern about the safety of its use in combination with other techniques that require metal electrodes implanted in the brain, such as electrocorticography (ECoG). The ECoG technique uses electrodes placed directly on the exposed surface of the brain to record electrical activity from the cerebral cortex during neurosurgical procedures. For the past five decades since the first electrocorticographs (Feindel and Penfield 1954), intraoperative ECoG has been used to assess and diagnose various epileptic form discharges in the cortex, as well as to assess eloquent cortex in brain tumor patients. The increasingly frequent application of both TMS and ECoG for neurological diagnostic and therapeutic purposes inevitably creates cases where the two techniques are used in parallel on a single subject. The lack of solid assessments of the potential hazards of this co-application is a problem that warrants careful investigation.

In this study, we evaluate the theoretical hazards of exposing cortical electrodes to TMS fields. The ECoG electrodes, being placed directly on the exposed surface of the cortex, are in a delicate position because any excessive electrode movement resulting from exposure to strong magnetic forces could cause irreversible damage to the brain tissue. It is therefore important to determine the safety of TMS applied in the presence of implanted electrodes.

Here we first briefly review the electromagnetic mechanisms pertinent to the problem: we can divide the sequence of actions into two well-known phenomena. The first, Faraday's law, states that time-varying magnetic fields induce electrical currents in adjacent conductive objects. During this phase, the alternating magnetic field $\vec{B}(t)$ which is itself produced by the alternating electric current $\mathrm{I}(\mathrm{t})$ in the TMS coil, induces eddy currents $\vec{J}(t)$ in implanted electrodes which are made of conducting material. The magnitude of this induced current is proportional to the rate of change of the magnetic field, which is in turn proportional to the rate of change of the current flow in the TMS coil. The second phenomenon is based on the Lorentz force law, which states that moving charges (and consequently, current-carrying objects) in the presence of magnetic fields are subject to magnetic forces. In other words, the 


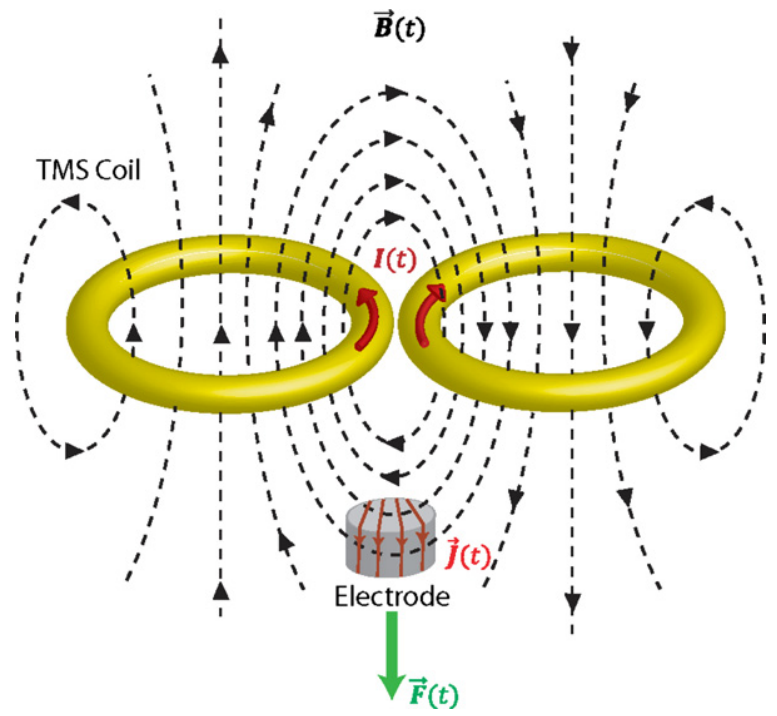

Figure 1. The schematic of ECoG electrode in the presence of TMS coil.

implanted electrode carrying the induced electric currents is now subject to a magnetic force $\vec{F}(t)$ with the amplitude and direction determined by the magnetic field of the stimulating coil (see figure 1). These electromagnetic forces tend to push the electrode toward the brain and thus induce mechanical stress in the brain tissue in contact with the electrode. Whether this causes irreversible damage to brain tissue is a question that can be answered only by analyzing the stress field inside the brain tissue in the vicinity of the electrode due to the external forces.

Consequently, our approach is first to compute the maximum magnetic forces imposed over a typical implanted electrode, and then to compare the induced mechanical stresses in the brain tissue to the maximum stress tolerable by the brain tissue. Considering the nature of the problem and the expected range of the electromagnetic forces (a few tens of milliNewton), the mechanical behavior of the brain under such loading conditions is modeled as an isotropic elastic medium. The equivalent stress induced due to the applied force is compared to the damage stress threshold reported in literature (Franceschini et al 2006). The ramifications of these calculations are then discussed.

\section{Materials and methods}

\subsection{Theoretical background}

Magnetic stimulation during TMS is based on Faraday's law of electromagnetic induction. When a conductive material is exposed to a time varying magnetic field, an electric field is induced which drives currents in the material. To calculate these induced currents, the timevarying magnetic field distribution inside the structure has to be evaluated. The behavior of electromagnetic fields in an arbitrary complex medium is governed by Maxwell's equations:

$$
\begin{aligned}
\nabla \times \vec{H} & =\vec{J}+\partial(\varepsilon \vec{E}) / \partial t \\
\nabla \times \vec{E} & =-\partial(\mu \vec{H}) / \partial t
\end{aligned}
$$


where $\vec{E}$ and $\vec{H}$ represent electric field strength $(\mathrm{V} / \mathrm{m})$ and magnetic field strength $(\mathrm{A} / \mathrm{m})$ respectively, while $\varepsilon$ and $\mu$ are permittivity $(\mathrm{F} / \mathrm{m})$ and permeability $(\mathrm{H} / \mathrm{m})$ of the medium and $\vec{J}$ is the electric current density $\left(\mathrm{A} / \mathrm{m}^{2}\right)$. The current density $\vec{J}$ inside the stimulating coil is assumed to be uniform with a magnitude determined by the power of the TMS pulse generator. This current is the primary source of electromagnetic fields in the system. By solving equation (1), the distribution of electric and magnetic fields can be obtained at any arbitrary location inside the head. Consequently, induced currents are conveniently calculated at each point as $\vec{J}=\sigma \vec{E}$, where $\sigma(\mathrm{S} / \mathrm{m})$ represents electrical conductivity of the medium. Once the induced fields and current densities are calculated at any point including in the implanted electrodes, the time averaged magnetic force imposed upon the electrode is straightforward to compute according to the Lorentz's law:

$$
\vec{F}_{m}=\frac{1}{2} \int_{v} \operatorname{Re}\left[\vec{J} \times \vec{B}^{*}\right] \mathrm{d} v
$$

where $\vec{B}(t)$ is the magnetic field at the location of the electrode with the superscript star $\left(^{*}\right)$ indicating the complex conjugate, $\vec{J}$ is the induced current density in the electrode and the integration is calculated over the electrode's volume.

There are important considerations in building a reliable electromagnetic model of TMS: first, although the quasi-static formulation has been extensively used to model TMS in the past (Amassian et al 1992, Branston and Tofts 2000, Eaton 1992, Esselle and Stuchly 1992, Roth et al 1991), recent studies have demonstrated that the ultra-high values of permittivity of biological tissues at low frequencies give rise to displacement currents which are comparable with ohmic currents (Wagner et al 2008, Starzynski et al 2002, Wagner et al 2004a, 2004b). In other words, if the reported extreme values of $\varepsilon_{r}\left(\approx 10^{7}\right)$ (Foster and Schwan 1996, Hart and Dunfree 1993, Hart et al 1996) are valid at low frequencies, $\sigma$ can be much less than $\omega \varepsilon$ or on the same order of magnitude and the simple quasi-static formulation will not be sufficiently accurate to give reliable results inside the head, as propagation effects become more important. Under these circumstances, it is possible that the presence of high-permittivity tissue alters the field distribution at the location of the electrode, leading to modified induced currents and affecting the magnitude of applied forces. This hypothesis was tested by comparing the results of free space simulations with those including the head model.

It is also important to note that although imposed magnetic forces were found to be in the order of milli Newtons, when applied on the very small surface of the electrode $\left(\sim 12 \mathrm{~mm}^{2}\right)$ these forces can lead to local pressures on the order of a few hundred Pascal. Considering the repetitive nature of most TMS applications (conventional rTMS pulses are composed of $\sim 10$ bursts per second) the possibility of local tissue damage cannot be safely refuted without performing a mechanical assessment of brain tissue in the vicinity of the electrode. Mechanical behavior of brain tissue is one of the most complicated to model among biological tissues. Viscoelastic (Galford and McElhaney 1970, Miller 1999, Sivaloganathan et al 2005a), poroelastic (Kaczmarek et al 1997, Sivaloganathan et al 2005b, Tenti et al 1999) and even purely elastic (Hakim et al 1976, Momjian and Bichsel 2006) models have been used in different analyses for various loading conditions. The characteristic time scale of force application is the important factor in choosing the material model. High strain rate deformation usually is modeled with viscoelasticity, whereas processes with low strain rate can be modeled using poroelasticity or mixture theory. In the case of the rapidly applied ( $<1 \mathrm{~ms}$ ) forces during TMS, the mechanical behavior of the brain tissue can be modeled as an isotropic and linearly viscoelastic medium (Franceschini et al 2006, Hrapko et al 2008, Laksari et al 2012, Prevost et al 2011). Due to such a short loading time period, the interstitial fluid (ISF) component of brain tissue does not have enough time to flow and the brain tissue behaves as nearly elastic 
and incompressible. Therefore the Poisson's ratio as an indication of volumetric deformability is very close to 0.5 ( $v \approx 0.499$ ) under these conditions (Franceschini et al 2006).

Mechanical behavior of an isotropic and linearly elastic medium is expressed via its shear modulus, in addition to the Poisson's ratio. The shear modulus of brain tissue has been reported from 300 to $6000 \mathrm{~Pa}$ for different brain regions, different ages, and different force application frequencies (Franceschini et al 2006, Hrapko et al 2008, Laksari et al 2012, Prevost et al 2011). The effect of loading conditions, such as temperature, loading direction, and precompression, are also known to affect the obtained shear modulus (Hrapko et al 2008, Chatelin et al 2011). In case of short duration pulses in our study $(<1 \mathrm{~ms})$, we chose the shear modulus of 6000 $\mathrm{Pa}$ as an upper limit as previously measured (Prevost et al 2011). Sensitivity of the calculated stresses to the shear modulus will be discussed later.

Considering the estimated shear modulus and Poisson's ratio under the determined loading, the stress field inside brain tissue under the electrode can be calculated. Because the volumetric deformation of brain tissue is negligible $(v \approx 0.499)$, shear stresses are considered the major potential cause of fiber matrix detachment (Franceschini et al 2006). Thus, our approach in this work was to compare the imposed shear stresses due to electromagnetic forces with stress thresholds which the brain tissue can bear without being damaged or distorted.

In summary, the first step to assess the safety of TMS applied on patients with implanted electrodes is to solve equations (1) to compute magnetic field produced by the TMS coil, as well as induced electric currents in the implanted electrode. In the next step, magnetic forces imposed over the electrode are computed using equation (2) for different relative position/orientation of electrode and TMS coil. The peak of this computed magnetic force is then used as the load in the mechanical analyses to evaluate the tissue stress in the worst case scenario. The details of these steps are provided in the following subsections.

\subsection{Electromagnetic analysis}

We used the Ansoft Maxwell 3D package which applies the finite element method (FEM) to solve a modified $T-\Omega$ formulation (Ansoft Ansys Product Suit 2011) of Maxwell's full-wave equations of (1). In this approach, divergence equations (Gauss laws) are automatically taken into account and used as accuracy check of the numerical method. The solution process was set with a default background boundary condition. 'Background' in Maxwell-3D is the region that surrounds the geometric model and fills any space that is not occupied by an object. The default boundary condition applied to the outmost surface of this region for the eddy current solver is the Neumann boundary condition (Hnormal $=0)$. The FE solver was set to follow an adaptive iterative process with the convergence limits determined by the energy error in the system. For each simulation, the mesh was refined between two successive iterations until the global energy error was less than $1 \%$.

2.2.1. Effect of high-permittivity head model. To investigate how the presence of adjacent high-permittivity tissue affects the distribution of induced currents inside the electrode and consequently alters the magnitude of applied forces, we compared three modeling scenarios: (1) TMS coil and electrode interacted through free space, (2) a realistic head model was included in the simulation with intermediate values of tissue relative permittivities $\left(\sim 10^{4}\right)$ and (3) a realistic head model was included in the simulation with extreme values of tissue relative permittivities $\left(\sim 10^{7}\right)$.

An anatomic head model was created using Amira, a 3D visualization and volume modeling system (Indeed-Visual Concepts TGS Inc. 2011) with the sample data obtained from the public domain Internet data base Visible Human Project (NLM). Fifty (50) cross 


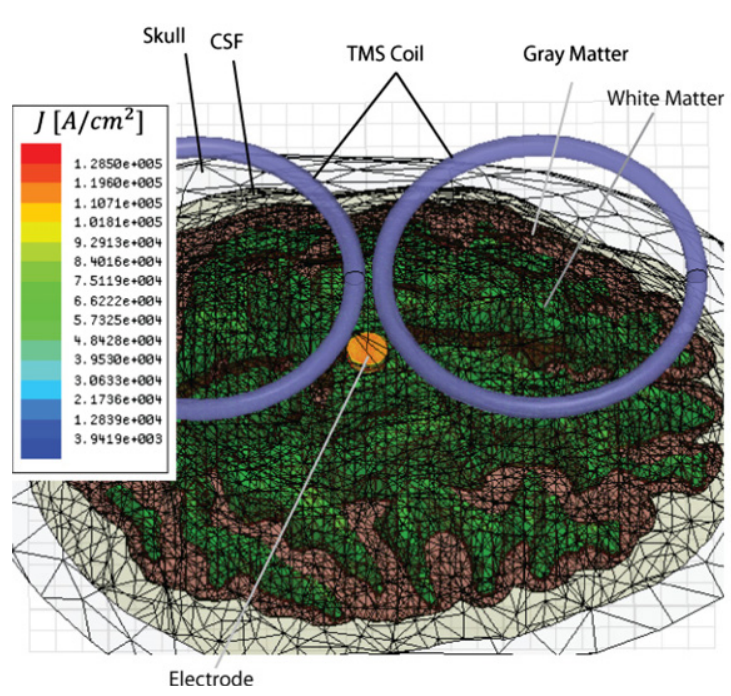

(a)

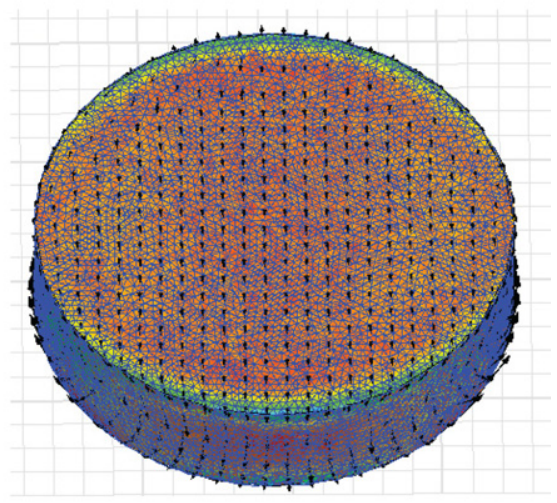

(b)

Figure 2. (a) The finite element model of brain consisting of skull, white matter, gray matter and CSF. A typical ECoG electrode is in direct contact with the surface of brain. The TMS coil is modeled as a figure-of-eight coil with its center positioned on top of the electrode. (b) The current distribution over the electrode. Mesh size was set to $<0.2 \mathrm{~mm}$ to accurately capture all the current loops.

Table 1. Electrical properties of tissues.

\begin{tabular}{llll}
\hline & $\begin{array}{l}\text { Conductivity } \\
\left(\mathrm{S} \mathrm{m}^{-1}\right)\end{array}$ & $\begin{array}{l}\text { Relative permittivity } \\
\text { (intermediate values) }\end{array}$ & $\begin{array}{l}\text { Relative permittivity } \\
\text { (extreme values) }\end{array}$ \\
\hline Bone cortical & 0.020 & $0.8 \times 10^{4}$ & $0.8 \times 10^{7}$ \\
Brain white matter & 0.066 & $1.2 \times 10^{4}$ & $1.2 \times 10^{7}$ \\
Brain gray matter & 0.109 & $1.2 \times 10^{4}$ & $1.2 \times 10^{7}$ \\
CSF & 2.000 & $0.6 \times 10^{4}$ & $0.6 \times 10^{7}$ \\
\hline
\end{tabular}

sections of the head of the Human Male data set were saved with in-plane resolution of $2048 \times 1216$ pixels $\left(0.3 \times 0.3 \mathrm{~mm}^{2}\right)$ and through-plane resolution of $1 \mathrm{~mm}$. The final 3D model after simplifications had an in-plane resolution of $2 \mathrm{~mm}$ and through-plane resolution of $4 \mathrm{~mm}$ and consisted of skull, CSF, white matter and gray matter (see figure 2). The construction process is similar to the method described in Golestanirad et al (2012) and Golestanirad et al (2010). The electrical conductivities of tissues were obtained from Niremf (2012). As stated, there is still considerable debate about the low frequency values of tissue permittivities; thus, we chose to allow for the inclusion of both intermediate values reported in literature and extreme values as predicted by existing alpha dispersion theories (Wagner et al 2004b). The electrical properties of tissues are summarized in table 1 .

The source was modeled as a figure-of-eight coil with two $3.5-\mathrm{cm}$-radius windings made of a single turn of 2-mm-radius copper wire. The copper was assumed to be a perfect conductor (no skin effect). The current in the coil was adjusted to produce a peak magnetic field of 2.2 T, as in Magstim Double $70 \mathrm{~mm}$ coil (model P/N 3190/1). The electrode was modeled as platinum ${ }^{7}$ with conductivity of $9.3 \times 106 \mathrm{~S} \mathrm{~m}^{-1}$ and permittivity and permeability set to

7 Typical EcoG electrodes, such as those manufactured by AD-TECH Medical Instrument Corporation are made of platinum. 


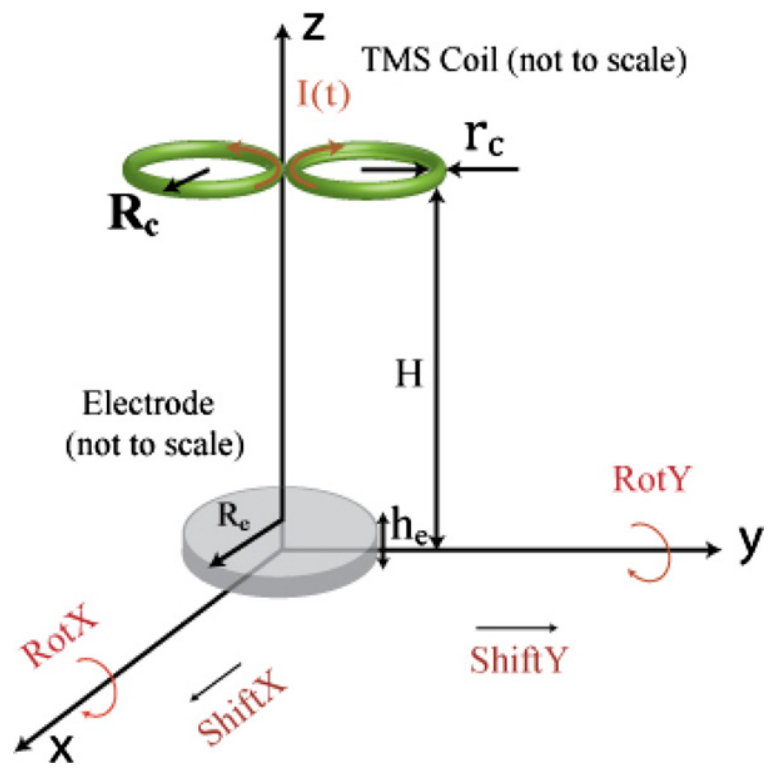

Figure 3. Simulation setup to investigate coil-electrode interaction in free space for different positions and orientations. TMS coil was fixed and the electrode was incrementally shifted and rotated along $x$ and $y$ axes. Dimensions are $r_{c}=2 \mathrm{~mm}, R_{c}=30 \mathrm{~mm}, H=20 \mathrm{~mm}, R_{e}=4 \mathrm{~mm}$ and $h_{e}=2 \mathrm{~mm}$. Current in the coil was set to $28 \mathrm{kA}$ which produced peak magnetic field of $2.2 \mathrm{~T}$.

$\varepsilon_{0}=8.854 \times 10^{-12}$ and $\mu_{0}=1.256 \times 10^{-6}$, respectively. Note that in clinical applications, TMS coil is positioned touching the surface of the head. However, commercially available coils have a 5-10 mm thick insulating layer covering the copper core (Jalinous 1991). The electrode itself is implanted at least $10-15 \mathrm{~mm}$ under the surface of skull. Therefore, we included a $20 \mathrm{~mm}$ gap between the electrode and the TMS coil in all simulation scenarios.

2.2.2. Effect of electrode-coil relative orientation/position. As demonstrated in section 3.1, presence of the head phantom in the model does not affect the magnitude of applied forces on the electrode in the frequency range of conventional TMS applications. This allowed us to perform the rest of electromagnetic simulations in free space, which significantly reduced the computational burden.

In clinical practice, TMS coil may be held with different orientations on the scalp. To investigate the effect of relative position and orientation of TMS coil and electrode on the magnitude of applied forces, we set up four simulation configurations as illustrated in figure 3 . In each simulation the electrode was incrementally displaced along one of the axes ( $x$ or $y)$ and rotated along either $\hat{x}$ or $\hat{y}$ direction.

Potential brain damage can happen when an excessive force is applied perpendicular to the surface of the electrode (toward the brain tissue) as the force component tangential to the brain surface, although could potentially misplace the electrode, will not cause damage to the tissue. Thus, in each case we calculated the component of magnetic force in the direction toward the brain tissue (perpendicular to electrode surface, away from TMS coil).

Solutions were obtained for the source at $5 \mathrm{kHz}$, with the current in the coil adjusted to produce the maximum magnetic field of $2.2 \mathrm{~T}$. The electrode was made of platinum with a 


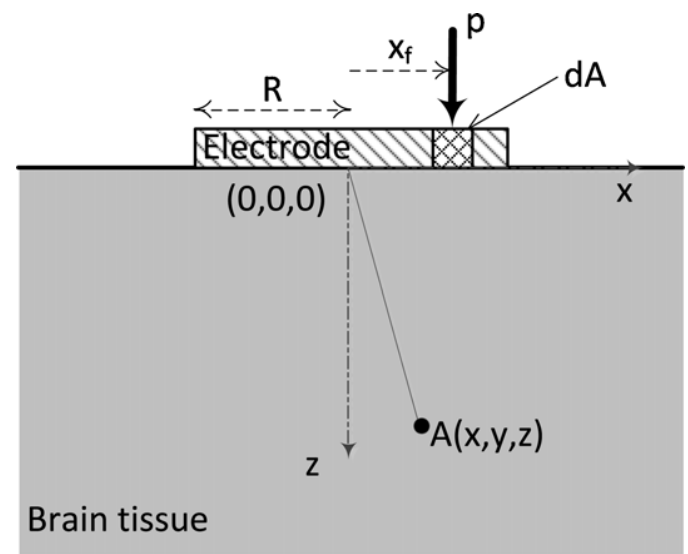

Figure 4. Calculation of the $3 \mathrm{D}$ stress tensor in brain tissue due to uniform electromagnetic force applied to the electrode on the brain surface.

radius of $R_{\mathrm{e}}=4 \mathrm{~mm}$ and a height of $h_{\mathrm{e}}=2 \mathrm{~mm}$ and its shortest distance from the center of TMS coil was $H=20 \mathrm{~mm}$.

2.2.3. Effect of electrode size. Typical ECoG electrodes have a radius of $2-4 \mathrm{~mm}$. However, to extend the results of this study to other potential applications, we have investigated the effect of electrode's size on computed forces by letting the radius of electrode vary from 1 to $10 \mathrm{~mm}$. To account for the worst case scenario (see section 3.2), simulations were performed for the case of electrode located $5 \mathrm{~cm}$ off center and rotated $135^{\circ}$ around $x$ direction, which corresponds to the peak of magnetic force.

2.2.4. Mechanical model of brain damage analysis. As discussed earlier, according to the short duration of the applied force, we used isotropic elastic behavior for the brain tissue under the electrode, characterized by the appropriate shear modulus and Poisson's ratio. Since the diameter of the brain is much larger than that of the electrode, the brain was modeled as a semi-infinite planar medium with the electrode in direct contact with its surface (see figure 4). Therefore, the stress field induced in the brain tissue due to the force exerted by the electrode could be accurately modeled based on 3D analytical solution of the stress field in a semi-infinite homogeneous elastic body subjected to normal pressure applied on its surface (Amenzade 1979). To do this, the electromagnetic force was modeled as a uniform pressure applied by the electrode to the brain at its contact surface. In figure 4, the stress tensor $(\sigma)$ at coordinate $(x, y, z)$ inside the brain tissue due to the electromagnetic force $(p)$ applied on an infinitesimal surface element $(\mathrm{d} A)$ at $(x f, y f, 0)$ is obtained as follows:

$$
\sigma(p)=\left[\begin{array}{lll}
\sigma_{x x}(p) & \sigma_{x y}(p) & \sigma_{x z}(p) \\
\sigma_{x y}(p) & \sigma_{y y}(p) & \sigma_{y z}(p) \\
\sigma_{x z}(p) & \sigma_{y z}(p) & \sigma_{z z}(p)
\end{array}\right]
$$

with

$\sigma_{x x}(p)=-\frac{p z}{2 \pi r^{3}}\left(3\left(\frac{x^{\prime}}{r}\right)^{2}-\frac{\mu}{\mu+\lambda}\right)-\frac{p \mu}{2 \pi(\mu+\lambda)}\left(\frac{y^{\prime 2}+z^{2}}{r^{3}(r+z)}-\frac{x^{\prime 2}}{r^{2}(r+z)^{2}}\right)$ 


$$
\begin{gathered}
\sigma_{y y}(p)=-\frac{p z}{2 \pi r^{3}}\left(3\left(\frac{y^{\prime}}{r}\right)^{2}-\frac{\mu}{\mu+\lambda}\right)-\frac{p \mu}{2 \pi(\mu+\lambda)}\left(\frac{x^{\prime 2}+z^{2}}{r^{3}(r+z)}-\frac{y^{\prime 2}}{r^{2}(r+z)^{2}}\right) \\
\sigma_{z z}(p)=-\frac{p z}{2 \pi r^{3}}\left(3\left(\frac{z}{r}\right)^{2}+\frac{\mu}{\mu+\lambda}\right)-\frac{p \mu}{2 \pi(\mu+\lambda)} \frac{z}{r^{3}} \\
\sigma_{x y}(p)=-\frac{3 p x^{\prime} y^{\prime} z}{2 \pi r^{5}}+\frac{p \mu}{2 \pi(\mu+\lambda)} \frac{x^{\prime} y^{\prime}(z+2 r)}{r^{3}(z+r)^{2}} \\
\sigma_{y z}(p)=-\frac{p y^{\prime}}{2 \pi r^{3}}\left(3\left(\frac{z}{r}\right)^{2}+\frac{\mu}{\mu+\lambda}\right)+\frac{p \mu}{2 \pi(\mu+\lambda)} \frac{y^{\prime}}{r^{3}} \\
\sigma_{x z}(p)=-\frac{p x^{\prime}}{2 \pi r^{3}}\left(3\left(\frac{z}{r}\right)^{2}+\frac{\mu}{\mu+\lambda}\right)+\frac{p \mu}{2 \pi(\mu+\lambda)} \frac{x^{\prime}}{r^{3}},
\end{gathered}
$$

where $r=\sqrt{\left(x^{\prime 2}+y^{\prime 2}+z^{2}\right)}, x^{\prime}=x-x_{f}, y^{\prime}=y-y_{f}, \mu=G, \lambda=G \frac{2 v}{1-2 v}$ and $G$ and $v$ are the shear modulus and Poisson's ratio. The coordinate origin is located at the center of the electrode. Because the total electromagnetic force $(F)$ is uniformly distributed over the electrode surface $(S)$, the elements of total stress $\left(\sigma^{T}\right)$ in coordinate of $(x, y, z)$ were calculated as follows:

$$
\sigma_{i j}^{T}=\int_{A} \sigma_{i j}\left(\frac{F}{S} \mathrm{~d} A\right), i, j=x, y, z .
$$

Then, for each coordinate of $(x, y, z)$, principal stresses $\left(\sigma_{1}, \sigma_{2}, \sigma_{3}\right)$ were calculated as eigenvalues of $\sigma^{T}$ and equivalent shear stress $\left(\sigma_{v}\right)$ was calculated as follows:

$\sigma_{v}=\sqrt{0.5\left(\left(\sigma_{x x}^{T}-\sigma_{y y}^{T}\right)^{2}+\left(\sigma_{y y}^{T}-\sigma_{z z}^{T}\right)^{2}+\left(\sigma_{z z}^{T}-\sigma_{x x}^{T}\right)^{2}+6\left(\sigma_{x y}^{T^{2}}+\sigma_{y z}^{T^{2}}+\sigma_{z x}^{T^{2}}\right)\right)}$.

Based on equations $(4 a)-(4 f)$, the shear modulus and consequently its variation with loading frequencies does not affect the stress tensor. However, Poisson's ratio affects the stress tensor. According to Franceschini et al (2006), we considered the undrained Poisson's ratio equal to 0.499 for the frequency range of conventional TMS pulses. To ensure the safe application of TMS, the values of $\sigma_{v}$ in all points inside the brain tissue in the vicinity of the electrode must be in the safe shear stress range. We calculated $\sigma_{v}$ in a grid of points extended to $150 \%$ of the radius of electrode inside the brain tissue, calculated the spatial maximum over these points and compared it with the maximum safe stress.

\section{Numerical results}

\subsection{Computing magnetic forces: effect of high-permittivity head model}

Figure 5 shows the magnitude of computed magnetic force applied on the electrode for three different modeling scenarios: (1) TMS coil and electrode interacting through free-space; (2) a realistic head model included in the model, with intermediate values of tissue relative permittivities in the order of $10^{4}$ (see table 1 for details) and (3) a realistic head model included in the model, with extreme values of tissue relative permittivities in the order of $10^{7}$. For all cases, simulations were performed sweeping a frequency range of 0-200 kHz.

It is demonstrated that for frequencies below $80 \mathrm{kHz}$, the presence of head model does not affect the magnitude of computed magnetic force applied on the electrode. However, at higher frequencies, the wavelength becomes comparable with the head dimensions $(\lambda / 2 \approx 20 \mathrm{~cm}$ at 


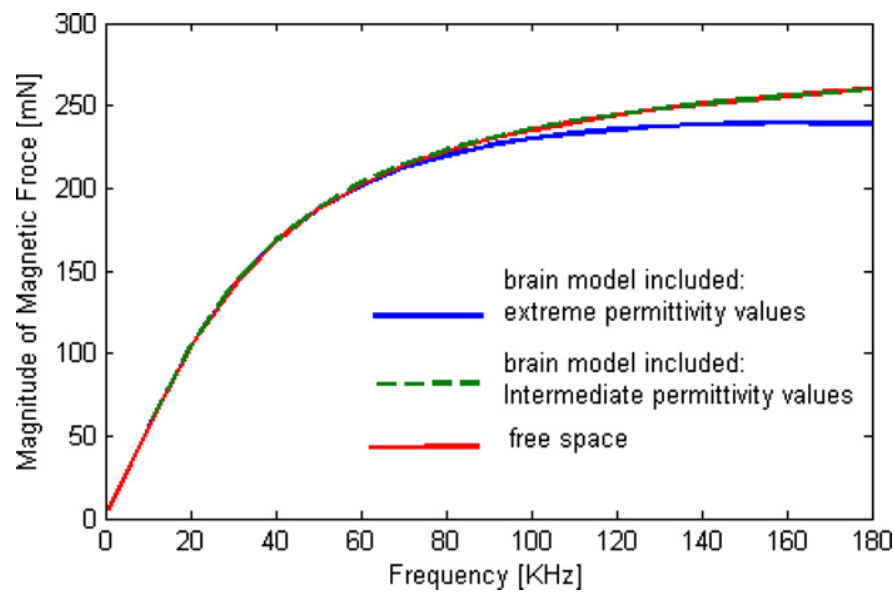

Figure 5. The magnitude of applied force on the implanted electrode for: (1) TMS coil and electrode interacting through free-space with no head model included in simulation (red line); (2) a realistic head model included in simulations, with relative tissue permittivities set to an intermediate level in the order of $\sim 10^{4}$ (dashed green line) and (3) a realistic head model included in simulations with relative tissue permittivities set to extreme values in order of $10^{7}$ (blue line).
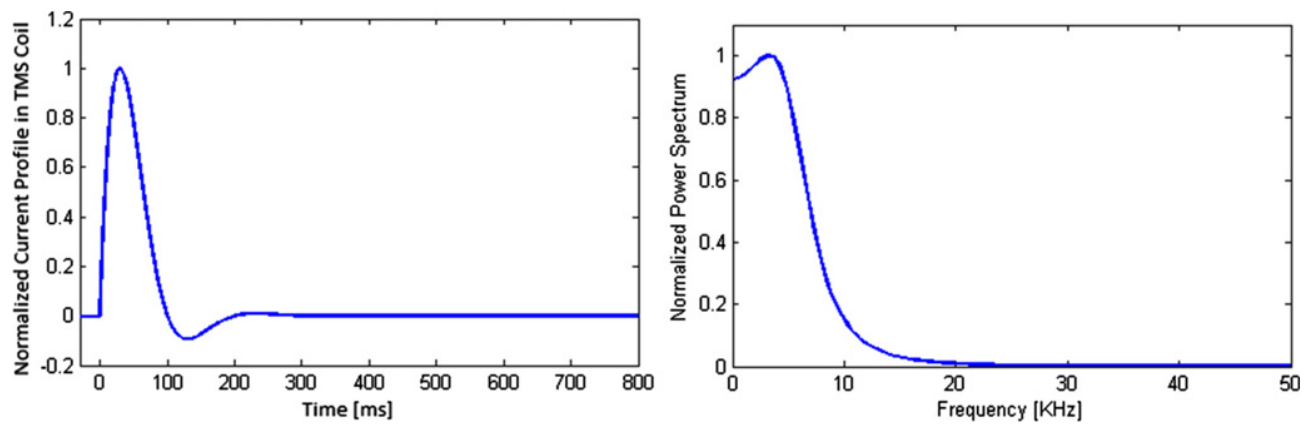

Figure 6. Left: time profile of electric current in TMS coil for Magstim 200 monophasic pulse. Right: power spectrum of monophasic pulse, computed using fast Fourier transform.

$200 \mathrm{kHz}$ for $\varepsilon_{r} \sim 10^{-7}$ ) and propagation effects modify the distribution of fields inside the head including at the location of the electrode.

The current pulse in TMS coil is generated when an initially charged capacitor $\mathrm{C}$, is discharged through the coil, with its inductance $\mathrm{L}$ and its resistance $\mathrm{R}$. The time dependence of the current in the coil is then (Kowalski et al 2002)

$$
i(t)=\frac{V_{0}}{\omega L} \mathrm{e}^{-\frac{\mathrm{Rt}}{2 L}} \sin \omega t,
$$

where $\omega=1 / \sqrt{\mathrm{LC}}$ is the resonant frequency of the circuit. Magstim 200 generates a monophasic pulse with a peak frequency component at approximately $5 \mathrm{kHz}$. An estimated time profile of the current pulse and its power spectrum is depicted in figure 6 based on equation (2) with values of R, L and C chosen to mimic Magstim 200 monophasic waveform (Sommer et al 2006). It is demonstrated that the frequency spectrum of the pulse does not have 


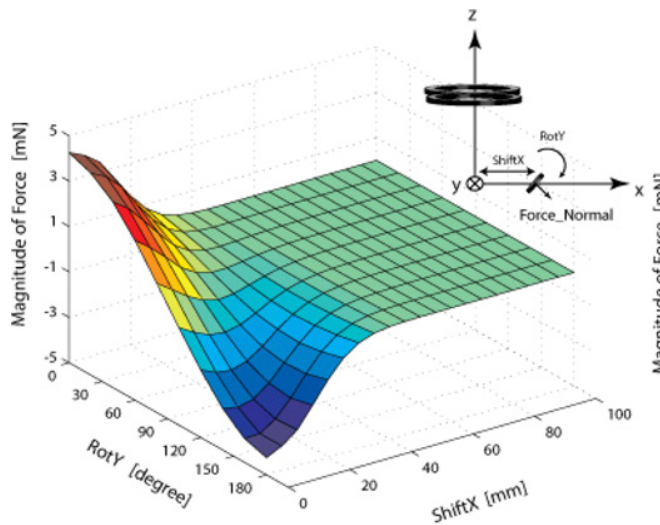

(a)

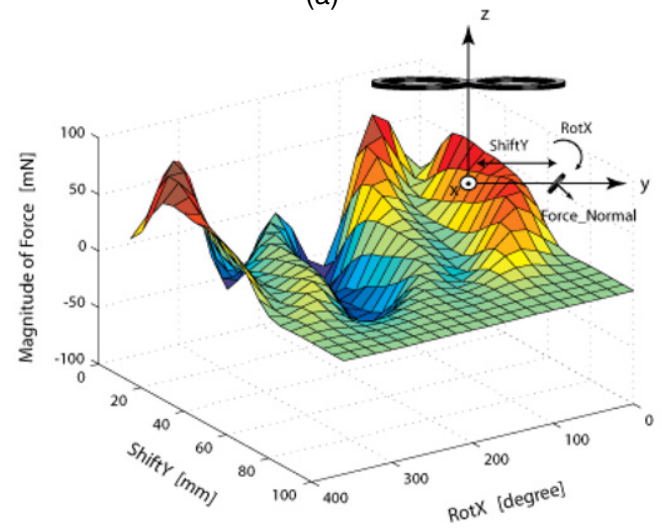

(c)

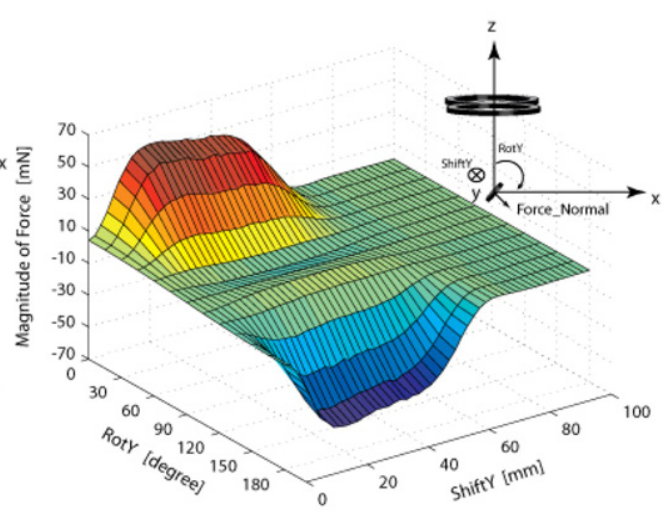

(b)

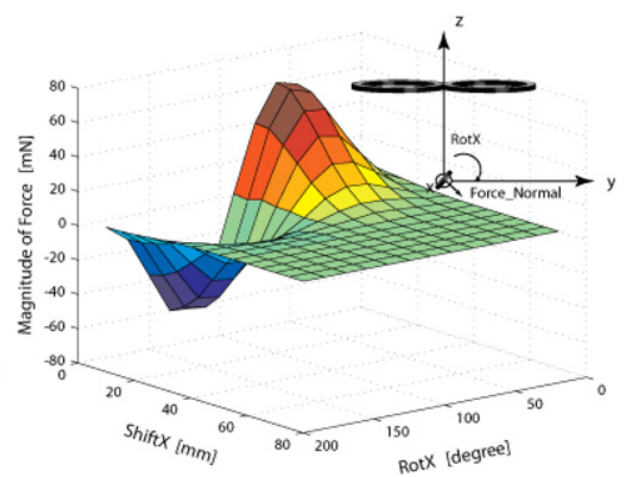

(d)

Figure 7. Magnitude of normal magnetic force as a function of (a) displacement along $x$ axis and rotation around $y$ direction, (b) displacement along $y$ axis and rotation around $y$ direction, (c) displacement along $y$ axis and rotation around $x$ direction and (d) displacement along $x$ axis and rotation around $x$ direction.

any significant component over $20 \mathrm{kHz}$. Therefore, free space modeling provides accurate results as long as we are interested in magnetic forces applied on the electrode.

\subsection{Computing magnetic forces: effect of electrode-coil relative orientation/position}

Figure 7(a) gives the distribution of magnetic force component perpendicular to the surface of electrode as a function of displacement along $x$-axis and rotation around $\hat{y}$ direction. The maximum of normal force in this case was found to be $\sim 4 \mathrm{mN}$ for electrode located under the center of TMS coil and parallel to its surface ( $\operatorname{ShiftX}=0$, RotY $=0$ ). For the case of electrode shifted along $y$ axis and rotated around $\hat{y}$ direction the maximum of normal force was formed at $18 \mathrm{~mm}$ off the center of TMS coil for the electrode parallel to the coil surface (ShiftY $=18 \mathrm{~mm}$, RotY $=0$ ) and reached its peak of $52 \mathrm{mN}$ (see figure 7(b)).

Figures $7(\mathrm{c})$ and (d) show the distribution of normal magnetic force when electrode was rotated around $\hat{x}$ direction and shifted along $x$ axis and $y$-axis, respectively. The worst case scenario corresponded to ShiftY $=5 \mathrm{~mm}$ and Rotx $=135^{\circ}$ with normal force reaching its peak of $70 \mathrm{mN}$. 


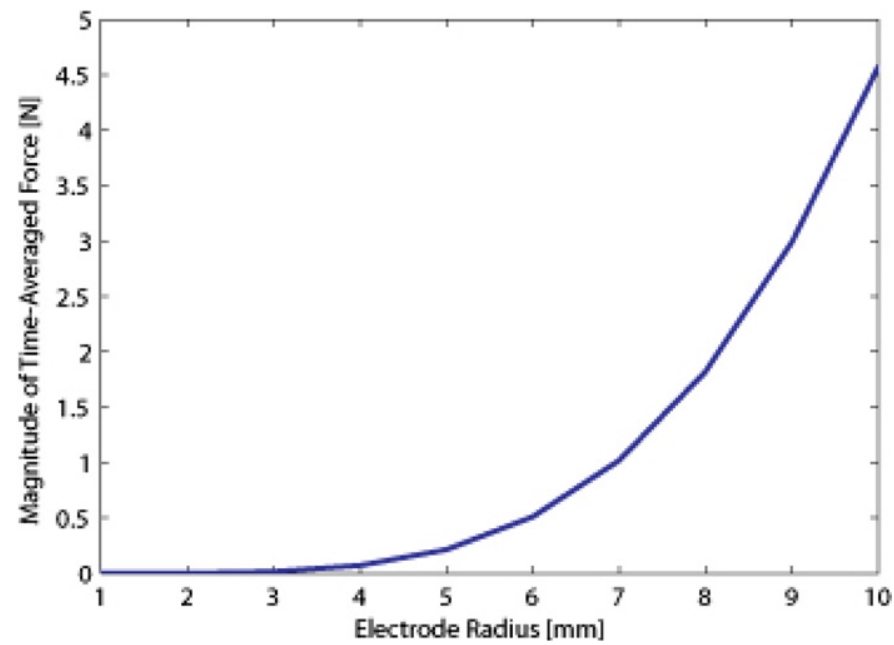

Figure 8. Magnitude of applied force as a function of electrode radius. Calculations were performed at $5 \mathrm{kHz}$ to account for the maximum force encountered during conventional TMS application. The electrode was positioned at the location of maximum magnetic force.

\subsection{Computing magnetic forces: effect of electrode size}

Figure 8 shows the magnitude of the normal component of time-averaged magnetic force applied on the electrode, as a function of electrode's radius. The height of electrode was set to $2 \mathrm{~mm}$ and it was positioned and oriented to receive the maximum force (ShiftX $=5 \mathrm{~mm}$, $\operatorname{RotX}=135^{\circ}$ ). Simulation was performed at $5 \mathrm{kHz}$ which is the peak component of the frequency spectrum of both monophasic and biphasic waveforms used in conventional TMS applications (Sommer et al 2006).

\subsection{Heating effects}

The ohmic loss associated with induced eddy currents in a conductive volume can be calculated as

$$
P_{0}=\frac{1}{2} \iiint_{v} R_{e}\left[\vec{E} \cdot \vec{J}^{*}\right] \mathrm{d} v
$$

where $\overrightarrow{J^{*}}$ is the complex conjugate of induced eddy current density, $\vec{E}$ is the electric field and the integration is computed over the volume of interest. The thermal energy associated with this ohmic loss can be computed as

$$
W=\tau P_{0},
$$

where $\tau$ will be the duration of TMS pulse in our case. The maximum $P_{0}$ was found to be $20 \mathrm{~W}$ which corresponds to $4 \mathrm{~mJ}$ dissipated energy for a $200 \mu$ s pulse. Changes in the temperature associated with the thermal energy dissipated in the electrode can then be calculated as

$$
\Delta T=\frac{W}{m C},
$$

where $\Delta T$ is change of temperature in Kelvin, $W$ is the thermal energy dissipated in the electrode, $m$ is electrode mass and $C$ is its specific heat capacity.

For the case of a platinum electrode $\left(C=0.13\left[\frac{\mathrm{kJ}}{\mathrm{kgK}}\right]\right)$ with a radius of $4 \mathrm{~mm}$ and height of $2 \mathrm{~mm}$ ( $m=2.2 \mathrm{~g})$ the change in the temperature will be $\sim 0.02 \mathrm{~K}$. Thus the heating effect can be considered negligible. 
Table 2. Equivalent shear stress and largest principal stress at $5 \mathrm{kHz}$ for different electrode radii.

\begin{tabular}{lll}
\hline Electrode radius $(\mathrm{mm})$ & Applied magnetic force $(\mathrm{N})$ & Equivalent shear stress $(\mathrm{Pa})$ \\
\hline 1 & $3.07 \mathrm{E}-05$ & $7.43 \mathrm{E}+00$ \\
2 & 0.0017 & $8.52 \mathrm{E}+01$ \\
3 & 0.0160 & $4.02 \mathrm{E}+02$ \\
4 & 0.0711 & $1.25 \mathrm{E}+03$ \\
5 & 0.2140 & $2.81 \mathrm{E}+03$ \\
6 & 0.5094 & $4.83 \mathrm{E}+03$ \\
7 & 1.0206 & $7.01 \mathrm{E}+03$ \\
8 & 1.8230 & $9.22 \mathrm{E}+03$ \\
9 & 2.9894 & $1.14 \mathrm{E}+04$ \\
10 & 4.5764 & $1.50 \mathrm{E}+04$ \\
\hline
\end{tabular}

\subsection{Damage assessment}

Table 2 gives the spatial maximum of distribution of the equivalent shear stress (over a grid of points in the vicinity of electrode) for different electrode radii. Franceschini et al (2006) reported that the brain tissue is not damaged under nominal stresses (uniaxial tension) below 2.71 (range: $1.28-7.10$ ) $\mathrm{kPa}$. The calculated equivalent shear stresses were found to be below the minimum tolerable stress $(1.28 \mathrm{kPa})$ for electrodes with a radius less than $4 \mathrm{~mm}$. On the other hand, the mechanical behavior of the brain tissue remains in the elastic zone under stresses below (insert symbol for 'approximately equal to') 300 Pa. Therefore, for the reported forces in table 2, with electrode radii below $2 \mathrm{~mm}$, the equivalent shear stress remains in the elastic zone. With larger stresses but still below $1.28 \mathrm{kPa}$ (electrode radii below $4 \mathrm{~mm}$ ), the brain tissue shows plastic deformation which is still safe according to Franceschini et al (2006). Nevertheless, cautions should be paid for such plastic deformations. Larger stresses may not be safe and are not recommended.

\section{Discussion and conclusion}

In this contribution we provided first steps toward safety assessment of force considerations applicable to TMS of patients with brain electrode implants. TMS alone or in combination with other electrophysiological techniques has been increasingly used to assess various pathologic conditions such as stroke, spinal cord injury, multiple sclerosis, Parkinson's disease and motor neuron disorders. Increasing application of TMS raises concern about the safety of its use in combination with other techniques that require metal electrodes implanted in the brain, such as ECoG. In this study, we evaluated the theoretical hazards of exposing cortical electrodes to TMS fields in two steps. First, a finite element electromagnetic analysis was performed to compute magnetic forces applied on implanted electrodes of different radii. Simulations were performed considering different relative positions and orientations of the electrode and TMS coil to compute the maximum value of magnetic forces applied on the electrode. Next, these computed forces were used in a mechanical model of the brain to compute the equivalent shear stress induced inside the brain tissue. These values are reported in table 2 and can be compared with the damage stress threshold reported in literature (approximately $2.7 \mathrm{kPa}$ ). The values reported in table 2 are calculated at $5 \mathrm{kHz}$, which is the peak frequency component of current conventional TMS pulses. Note that in low frequencies $(<30 \mathrm{kHz})$, the magnetic force is linearly increasing with frequency of the source. This is in accordance with analytical formulations describing the behavior of induced currents in simple geometries under the quasi-static regime, where the magnitudes of induced electric fields (and thus induced electric 
currents) are proportional to the rate of change of current $(\mathrm{d} I / \mathrm{d} t)$ in the TMS coil (see figure 2). Also note that values of force magnitude reported in table 2 are calculated for a peak magnetic field of 2.2 T (as in Magstim Double $70 \mathrm{~mm}$ coil). The force in general is proportional to the square of the magnetic field produced by TMS coil (see equation (2) and note that the induced current $\mathbf{J}$ itself is proportional to the magnetic field $\mathbf{B}$. Considering these facts, one can calculate the maximum force for different source frequencies and output fields:

$F_{m}\left(R_{e}, p, B, f\right)=F_{m}^{\prime}\left(R_{e}\right) \times p \times\left(\frac{B}{2.2}\right)^{2} \times \frac{f}{5} \cong 4.13 \times 10^{-2} F_{m}^{\prime}\left(R_{e}\right) p f B^{2}$

where $F_{m}\left(R_{e}, p, B, f\right)$ is the magnetic force (in Newton) applied on an electrode of a radius of $R_{e}$ (millimeters), when a TMS coil with maximum magnetic field of $B$ (Tesla) is used at $p$ percent of its output power. $f$ is the peak frequency component of the TMS pulse spectrum in $\mathrm{kHz}$ (computed for a single pulse as demonstrated in figure 6) and $F_{m}^{\prime}$ is the value of magnetic force (in Newton) reported in table 2.

To make use of larger electrodes safely, one could think of introducing microscopic grooves on the surface of electrode to suppress eddy currents and reduce both magnetic force and ohmic heating. Resulting electrodes may potentially be shown to be TMS compatible, although it is possible that the intervention affects the recording performance of the ECoG system.

\section{Acknowledgment}

This work was partially supported by research grants from Swiss National Science Foundation (grant numbers PBELP2-135868 and 137539).

\section{References}

Amassian V E, Eberle L, Maccabee P J and Cracco R Q 1992 Modelling magnetic coil excitation of human cerebral cortex with a peripheral nerve immersed in a brain-shaped volume conductor: the significance of fiber bending in excitation Electroencephalogr. Clin. Neurophysiol. Evoked Potentials 85 291-301

Amenzade Y A 1979 Theory of Elasticity (Moscow: Mir)

Ansoft Ansys Product Suit 2011 Maxwell 3D Available at: http://www.ansoft.com/products/em/maxwell/

Barker A T, Freeston I L, Jalinous R and Jarratt J A 1987 Magnetic stimulation of the human brain and peripheral nervous system: an introduction and the results of an initial clinical evaluation Neurosurgery 20 100-9

Branston N and Tofts P 2000 Analysis of the distribution of currents induced by a changing magnetic field in a volume conductor Phys. Med. Biol. 36161

Chatelin S, Vappou J, Roth S, Raul J and Willinger R 2011 Towards child versus adult brain mechanical properties J. Mech. Behav. Biomed. Mater. 6 166-73

Classen J, Wolters A, Stefan K, Wycislo M, Sandbrink F, Schmidt A and Kunesch E 2004 Paired associative stimulation Suppl. Clin. Neurophysiol. 57 563-9

Dimyan M and Cohen L 2010 Contribution of transcranial magnetic stimulation to the understanding of mechanisms of functional recovery after stroke Neurorehabil. Neural Repair 24 125-35

Eaton H 1992 Electric field induced in a spherical volume conductor from arbitrary coils: applications to magnetic stimulation and MEG Med. Biol. Eng. Comput. 30 433-40

Elahi B and Chen R 2009 Effect of transcranial magnetic stimulation on Parkinson motor function-systematic review of controlled clinical trials Mov. Disord. 24 357-63

Esselle K P and Stuchly M A 1992 Neural stimulation with magnetic fields: analysis of induced electric fields IEEE Trans. Biomed. Eng. 39 693-700

Feindel W and Penfield W 1954 Localization of discharge in temporal lobe automatism Arch. Neurol. Psychiatry 72 605-30

Foster K R and Schwan H P 1996 Dielectric properties of tissues Handbook of Biological Effects of Electromagnetic Fields ed C Polk and E Postow (Boca Raton, FL: CRC Press) pp 25-102

Franceschini G, Bigoni D, Regitnig P and Holzapfel G A 2006 Brain tissue deforms similarly to filled elastomers and follows consolidation theory J. Mech. Phys. Solids 54 2592-620 
Galford J E and McElhaney J H 1970 A viscoelastic study of scalp, brain, and dura J. Biomech. 3 211-21

Golestanirad L, Izquierdo A P, Graham S J, Mosig J R and Pollo C 2012 Effect of realistic modeling of deep brain stimulation on the prediction of volume of activated tissue Prog. Electromagn. Res. 126 1-16

Golestanirad L, Mattes M, Mosig J R and Pollo C 2010 Effect of model accuracy on the result of computed current densities in the simulation of transcranial magnetic stimulation IEEE Trans. Magn. 46 4046-51

Hakim S, Venegas J G and Burton J D 1976 The physics of the cranial cavity, hydrocephalus and normal pressure hydrocephalus: mechanical interpretation and mathematical model Surg. Neurol. 5 187-210

Hart F X and Dunfree W R 1993 In vivo measurements of low frequency dielectric spectra of a frog skeletal muscle Phys. Med. Biol. 38 1099-112

Hart F X, Toll R B and Berner N J 1996 The low frequency dielectric properties of octopus arm muscle measured in vivo Phys. Med. Biol. 41 2043-52

Hrapko M, Van Dommelen J, Peters G and Wismans J 2008 The influence of test conditions on characterization of the mechanical properties of brain tissue J. Biomech. Eng. 130031003

Ilmoniemi R J, Ruohonen J and Karhu J 1999 Transcranial magnetic stimulation — a new tool for functional imaging of the brain Crit. Rev. Biomed. Eng. 27 241-81

Indeed-Visual Concepts TGS Inc. 2011 Amira 5.2-User's Guide and Reference Manual Available at: http://www.amira.com/

Jalinous R 1991 Technical and practical aspects of magnetic nerve stimulation J. Clin. Neurophysiol. 810

Kaczmarek M, Subramaniam R and Neff S 1997 The hydromechanics of hydrocephalus: steady-state solutions for cylindrical geometry Bull. Math. Biol. 59 295-323

Kowalski T, Silny J and Buchner H 2002 Current density threshold for the stimulation of neurons in the motor cortex area Bioelectromagnetics 23 421-8

Kujirai T, Caramia M D, Rothwell J C, Day B L, Thompson P D, Ferbert A, Wroe S, Asselman P and Marsden C D 1993 Corticocortical inhibition in human motor cortex J. Physiol. 471 501-19

Laksari K, Shafieian M and Darvish K 2012 Constitutive model for brain tissue under finite compression J. Biomech.

Miller K 1999 Constitutive model of brain tissue suitable for finite element analysis of surgical procedures J. Biomech. 32 531-7

Momjian S and Bichsel D 2006 Elastic and poro-elastic models of ventricular dilatation in hydrocephalus Proc. COMSOL Users Conf. (Grenoble, 2006)

Niremf 2012 Calculation of the Dielectric Properties of Body Tissues Available: http://niremf.ifac.cnr.it

NLM 2011 The visible human project(Online) Available: http://www.nlm.nih.gov/research/visible/visible_human.html

Nowak D A, Bösl K, Podubeckà J and Carey J R 2010 Noninvasive brain stimulation and motor recovery after stroke Restor. Neurol. Neurosci. 28 531-44

Pascual-Leone A, Walsh V and Rothwell J 2000 Transcranial magnetic stimulation in cognitive neuroscience-virtual lesion, chronometry, and functional connectivity Curr. Opin. Neurobiol. 10 232-7

Prevost T P, Balakrishnan A, Suresh S and Socrate S 2011 Biomechanics of brain tissue Acta Biomater. 7 83-95

Rossini P M and Rossi S 2007 Transcranial magnetic stimulation: diagnostic, therapeutic, and research potential Neurology 68 484-8

Roth B J, Saypol J M, Hallett M and Cohen L G 1991 A theoretical calculation of the electric field induced in the cortex during magnetic stimulation Electroencephalogr. Clin. Neurophysiol. Evoked Potentials 81 45-76

Sivaloganathan S, Stastna M, Tenti G and Drake J M 2005a Biomechanics of the brain: a theoretical and numerical study of Biot's equations of consolidation theory with deformation-dependent permeability Int. J. Non-Linear Mech. 40 1149-59

Sivaloganathan S, Stastna M, Tenti G and Drake J M 2005b A viscoelastic approach to the modelling of hydrocephalus Appl. Math. Comput. 163 1097-107

Sommer M, Alfaro A, Rummel M, Speck S, Lang N, Tings T and Paulus W 2006 Half sine, monophasic and biphasic transcranial magnetic stimulation of the human motor cortex Clin. Neurophysiol. 117 838-44

Starzynski J, Sawicki B, Wincenciak S, Krawczyk A and Zyss T 2002 Simulation of magnetic stimulation of the brain IEEE Trans. Magn. 38 1237-40

Tenti G, Sivaloganathan S and Drake J M 1999 Brain biomechanics: steady state consolidation theory of hydrocephalus CAMQ 7 93-110

Wagner T, Gangitano M, Theoret R R H, Kobayashe M, Anschel D, Ives J, Cuffin B N, Schomer D and PascualLeone A 2004a Intracranial measurement of current densities induced by transcranial magnetic stimulation in the human brain Neurosci. Lett. 354 91-4

Wagner T A, Zahn M, Grodzinsky A J and Pascual-leone A 2004b Three-dimensional head model simulation of transcranial magnetic stimulation IEEE Trans. Biomed. Eng. 51 1586-98

Wagner T, Rushmore J, Eden U and Valero-cabre A 2008 Biophysical foundations underlying TMS: setting the stage for an effective use of neurostimulation in the cognitive neurosciences Cortex 45 1025-34 